\title{
Crystal structure of the NADP+ ${ }^{+}$-dependent aldehyde dehydrogenase from Vibrio harveyi: structural implications for cofactor specificity and affinity
}

\author{
Bijan AHVAZI ${ }^{1}$, Rene COULOMBE${ }^{1}$, Marc DELARGE ${ }^{1}$, Masoud VEDADI, Lei ZHANG, Edward MEIGHEN and Alice VRIELINK ${ }^{1,2}$ \\ Biochemistry Department, McIntyre Medical Sciences Building, McGill University, 3655 Drummond Street, Montréal, Québec, Canada H3G 1 Y6
}

\begin{abstract}
Aldehyde dehydrogenase from the bioluminescent bacterium, Vibrio harveyi, catalyses the oxidation of long-chain aliphatic aldehydes to acids. The enzyme is unique compared with other forms of aldehyde dehydrogenase in that it exhibits a very high specificity and affinity for the cofactor $\mathrm{NADP}^{+}$. Structural studies of this enzyme and comparisons with other forms of aldehyde dehydrogenase provide the basis for understanding the molecular features that dictate these unique properties and will enhance our understanding of the mechanism of catalysis for this class of enzyme. The X-ray structure of aldehyde dehydrogenase from $V$. harveyi has been solved to $2.5-\AA$ resolution as a partial complex with the cofactor $\mathrm{NADP}^{+}$and to $2.1-\AA$ resolution as a fully bound 'holo' complex. The cofactor preference exhibited by different forms of the enzyme is predominantly determined by the electrostatic environment surrounding the 2 '-hydroxy or
\end{abstract}

the $2^{\prime}$-phosphate groups of the adenosine ribose moiety of $\mathrm{NAD}^{+}$ or $\mathrm{NADP}^{+}$, respectively. In the $\mathrm{NADP}^{+}$-dependent structures the presence of a threonine and a lysine contribute to the cofactor specificity. In the $V$. harveyi enzyme an arginine residue (Arg210) contributes to the high cofactor affinity through a pi stacking interaction with the adenine ring system of the cofactor. Further differences between the $V$. harveyi enzyme and other aldehyde dehydrogenases are seen in the active site, in particular a histidine residue which is structurally conserved with phosphorylating glyceraldehyde-3-phosphate dehydrogenase. This may suggest an alternative mechanism for activation of the reactive cysteine residue for nucleophilic attack.

Key words: cofactor binding, dehydrogenases, enzyme mechanism, nucleotide specificity.

\section{INTRODUCTION}

Aldehyde dehydrogenases constitute a large family of NAD $(\mathrm{P})^{+}$dependent enzymes found in both prokaryotes and eukaryotes. The enzyme catalyses the dehydrogenation of aldehydes via the formation of a thiohemiacetal covalent intermediate with the aldehyde substrate. Although a few enzymes can use both $\mathrm{NADP}^{+}$ and $\mathrm{NAD}^{+}$, aldehyde dehydrogenase from the bioluminescent bacterium, Vibrio harveyi, shows a strong preference for $\mathrm{NADP}^{+}$ with a $K_{\mathrm{m}}$ over 150 -fold lower for $\mathrm{NADP}^{+}$than for $\mathrm{NAD}^{+}$[1].

In mammalian systems, three classes of aldehyde dehydrogenase have been identified on the basis of amino acid sequence and localization: class 1 (ALDH1), cytosolic; class 2 (ALDH2), mitochondrial; and class 3 (ALDH3), tumour-associated [2]. Variation in the quaternary structure has been observed between the different classes of the enzyme: the class- 1 and -2 enzymes exist as homotetramers of $50-55 \mathrm{kDa}$ subunits whereas the class3 form is a homodimer [3]. A few of these enzymes, particularly the class-3 subfamily, have been shown to exhibit a relatively weak interaction with the $\mathrm{NADP}^{+}$cofactor $\left(K_{\mathrm{m}}\right.$ values of $>$ $60 \mu \mathrm{M}$ [2]). Two $\mathrm{NADP}^{+}$-specific forms of aldehyde dehydrogenase have been identified: one from Streptococcus mutans and one from $V$. harveyi. In the case of the $V$. harveyi enzyme not only is a preference for $\mathrm{NADP}^{+}$observed but the affinity of the enzyme for the cofactor is unusually high. Byers and Meighen have reported a $K_{\mathrm{m}}$ of $1.4 \mu \mathrm{M}$ for $\mathrm{NADP}^{+}$with saturating dodecanal [1], whereas the $\mathrm{NADP}^{+}$-specific $S$. mutans enzyme has a $K_{\mathrm{m}}$ of $24.5 \mu \mathrm{M}$ for the cofactor [4].

The amino acid sequences of a large number of aldehyde dehydrogenases have been characterized with only 23 invariant amino acid residues [5]. Although the sequence similarity of the enzyme from $V$. harveyi with other aldehyde dehydrogenases is low $(18.5-22.5 \%$ identity, with only 19 of the 23 reported invariant residues present [6]), the $V$. harveyi enzyme appears to be most closely related to the class- 3 mammalian enzyme. This similarity is consistent with the observed dimeric structure of the $V$. harveyi enzyme as well as the kinetic properties and substrate specificities. Both enzymes exhibit higher $V_{\max } / K_{\mathrm{m}}$ values for increasing aldehyde chain length, suggesting the existence of a hydrophobic pocket at the active site that selects for longer-chain aldehydes [7-9].

Among the invariant amino acids there is only one conserved cysteine residue: Cys-302 from the class- 1 and -2 mammalian enzymes, Cys-243 from the class-3 enzyme and Cys-289 in the enzyme from $V$. harveyi. This residue is considered to be the most likely candidate for formation of the thiohemiacetal covalent intermediate with aldehyde in the active site of the enzyme [5,6,10,11]. Mutagenesis of this residue in $V$. harveyi to a serine results in a significant decrease in activity, without preventing coenzyme binding [6]. Aside from this cysteine residue two conserved glutamic acid residues (Glu-253 and Glu-377 in aldehyde dehydrogenase from $V$. harveyi) are invariant. Glu-253 has been implicated in acting as a general base in activation of the cysteine nucleophile for both the class- 2 and the $V$. harveyi enzymes [11,12]. Mutations of Glu-377 have led to the suggestion that this residue is involved in the hydride-transfer step [12]. In an attempt to identify residues important for cofactor interaction we have carried out mutagenesis and binding studies of the conserved GXXXXG fingerprint region (residues 229-234) in $V$. harveyi $[13]$. These studies have shown that the last glycine in the

Abbreviations used: ADPP, adenine, ribose and pyrophosphate portion; ALDH1, class-1 cytosolic aldehyde dehydrogenase; ALDH2, class-2 mitochondrial ALDH; ALDH3, class-3 tumour-associated ALDH; ALDH9, betaine ALDH; MAD, multiple wavelength anomalous dispersion; NCS, noncrystallographic symmetry.

1 These authors are members of the collaborative group known as the Montreal Joint Center for Structural Biology

2 To whom correspondence should be addressed (e-mail alice.vrielink@bri.nrc.ca). 
fingerprint (Gly-234) is not absolutely critical for nucleotide binding, whereas mutation of the first glycine (Gly-229) to alanine resulted in a decreased binding of both $\mathrm{NAD}^{+}$and $\mathrm{NADP}^{+}$.

Crystal structures have been determined for a number of $\mathrm{NAD}^{+}$-specific forms of aldehyde dehydrogenase [14-18] as well as an $\mathrm{NADP}^{+}$-specific form of the enzyme from $S$. mutans [19]. In this paper we report the structure of the $\mathrm{NADP}^{+}$-specific aldehyde dehydrogenase from $V$. harveyi. Structural comparisons between the $\mathrm{NAD}^{+}$- and $\mathrm{NADP}^{+}$-specific forms enable a detailed molecular comparison to be made and will provide information on the requirements that dictate cofactor specificity.

\section{EXPERIMENTAL}

\section{Crystallization}

Aldehyde dehydrogenase from $V$. harveyi was expressed and purified as outlined by Meighen and coworkers [6]. The protein was dialysed into $25 \mathrm{mM}$ Hepes, $\mathrm{pH} 7.0 / 10 \mathrm{mM} \beta$-mercaptoethanol in order to remove the $\mathrm{NADP}^{+}$cofactor from the enzyme. The partially bound $\mathrm{NADP}^{+}$-enzyme complex was crystallized as described previously [20]. Crystals for the 'holo' enzyme were obtained by adding $1.0 \mathrm{mM} \mathrm{NADP}^{+}$to the enzyme preparation. The complex was crystallized using the same procedure as for the partially bound complex. Crystals for both complexes belong to the monoclinic space group $\mathrm{P} 2{ }_{1}$ with cell parameters of $a=79.4 \AA, b=131.2 \AA, c=92.5 \AA$ and $\beta=$ $92.5^{\circ}$. The asymmetric unit contains four molecules. All data collection was carried out on crystals that were briefly soaked in $12 \%$ glycerol and then transferred to a stream of nitrogen at 80 $\mathrm{K}$ generated by an ADSC (Area Detector Systems Corporation) Low Temperature Crystal cooling system.

\section{Structure solution and refinement}

The partially bound complex crystal structure was solved by a combination of multiple isomorphous replacement and multiple wavelength anomalous diffraction (MAD) using a mutant of the enzyme in which the methionine residues were replaced with selenomethionine. The heavy-atom derivative data and the native data were collected using an imaging plate detector (MAR Research) mounted on a Rigaku RU-200 rotating-anode X-ray generator with a double-focusing mirror system (Supper). MAD data at three wavelengths were collected on a single selenomethionyl mutant crystal at beamline X12C (National Synchrotron Light Source, Brookhaven National Laboratory, New York, NY, U.S.A.) using a Brandeis CCD (charge-coupled device) area detector. The data for the fully occupied complex were collected at beamline X8C using a MAR image plate detector. The datacollection statistics are given in Table 1. The images collected for all data sets were processed and scaled using the HKL suite of software $[21,22]$. Further data analysis and heavy-atom refinement was carried out using the CCP4 suite of software [23].

Data collected on a crystal soaked in a solution of mother liquor containing $0.05 \mathrm{mM}$ mercuric acetate for $2.5 \mathrm{~h}$ produced a strongly isomorphous derivative with four mercury positions in the asymmetric unit. A second heavy-atom derivative was identified from difference Fourier maps on data from a crystal soaked in mother liquor containing $1 \mathrm{mM} \mathrm{K}_{2} \mathrm{PtCl}_{4}$. A third heavy-atom derivative was found using $1 \mathrm{mM}$ parachloromercuribenzenesulphonic acid resulting in different heavyatom occupancies from those of the mercuric acetate derivative. In addition, a selenomethionine mutant of the enzyme was expressed in the $\mathrm{Met}^{-}$strain using the procedure described by Hendrickson et al. [24]. Purification and crystallization of the mutant were carried out using the identical protocols to the native protein. The phases obtained from the heavy-atom derivatives were used to calculate anomalous and dispersive Fourier maps from which 23 of the expected 28 selenium positions could be located in the asymmetric unit. The refinement of the selenium as well as the mercury and platinum positions was carried out using the program MLPHARE treating the MAD data as a special case of multiple isomorphous replacement according the procedure outlined by Ramakrishnan and Biou [25]. The phases were optimized further by solvent flattening and histogram matching using the program DM, with a solvent content of $40 \%$. The non-crystallographic symmetry (NCS) matrices relating the four protomers in the asymmetric unit were determined using the heavy-atom and selenium positions within each protomer. A mask was built around a single protomer using the bones atoms in the program $\mathrm{O}$ [26] and 4-fold averaging performed with the RAVE software $[27,28]$. The electron-density map was clearly interpretable and enabled an initial model of 411 of the total 510 residues within a single protomer to be built using the program $\mathrm{O}$ [26] and refined with the program CNS [29].

Crystallographic refinement to $2.5-\AA$ resolution was carried out using the inflection point data of the selenomethionine mutant for the partially occupied complex. SIGMAA-weighted maps calculated with coefficients $3 \mathrm{Fo}-2 \mathrm{Fc}$ and $\mathrm{Fo}-\mathrm{Fc}$ were used for model rebuilds. As the refinement progressed, the adenine, ribose and pyrophosphate portion (ADPP) of the ligand became apparent in the electron-density map despite the fact that the protein had been dialysed prior to crystallization to remove the cofactor. In order to determine the occupancy of the cofactor in the enzyme we analysed for the presence of $\mathrm{NADP}^{+}$in protein that had been dialysed against buffer to remove the cofactor, in a fashion similar to the treatment of the enzyme prior to crystallization. These studies showed that after dialysis of the enzyme for 2-3 days approx. $50 \%$ of the nucleotide-binding sites contain $\mathrm{NADP}^{+}(\approx 1.0 \mathrm{~mol} /$ dimer; E. Meighen, unpublished work). On this basis, the occupancy of the ADPP was set to 0.5 during crystallographic refinement.

Initially constrained NCS symmetry was imposed during the refinement; however, in the later stages the NCS weights were set to 300 for all protein atoms and removed completely for regions of the structure involved in crystal contacts. NCS restraints were applied to the temperature factors during the refinement. Crossvalidation was carried out at all stages of the refinement and bulk solvent corrections were included in the final cycles. The final model includes residues 5-508 for one protomer within the asymmetric unit and residues 7-508 for the remaining three protomers. In addition, the final model for the tetramer includes 413 water molecules and one ADPP of the $\mathrm{NADP}^{+}$ligand for each protomer.

The refined co-ordinates for the $2.5-\AA$ partially occupied structure were used for the initial model of the fully occupied complex. Refinement was carried out to $2.1-\AA$ resolution with NCS restraints on positional parameters and temperature factors. The weights imposed for NCS restraints were 200 for main-chain atoms and 100 for side-chain atoms. Flexible loop regions of the structure were refined with the NCS weight set to 25 . The model rebuilds were carried out using a 4-fold averaged electron-density map. After the first cycle of refinement the full $\mathrm{NADP}^{+}$ligand was included in the model at full occupancy. The temperature factors for the nicotinimide and ribose moieties, however, are large indicating that there is significant conformational mobility for this region of the cofactor. This observation is consistent with previously determined structures of aldehyde dehydrogenases, all of which show poorly defined electron density for the nicotinamide and ribose moieties and supports the suggestion 
Table 1 Data collection and processing statistics

I/ $\sigma$ represents the signal-to-noise ratio; \% Complete refers to data completeness. PCMBS, para-chloromercuribenzenesulphonic acid; SeMet, selenomethionine.

\begin{tabular}{|c|c|c|c|c|c|c|}
\hline & Resolution $(\AA)$ & Total reflections & Independent reflections & $1 / \sigma$ & $\%$ Complete & $R_{\text {merge }}(\%)$ \\
\hline Native $1^{*} \uparrow$ & $2.8(2.9-2.8)$ & 105797 & 43715 & $16.8(2.5) \|$ & $94.0(68.0)$ & $6.9(19.7)$ \\
\hline Native $2 \ddagger$ & $2.1(2.2-2.1)$ & 189982 & 92467 & $24.9(17.2)$ & $83.9(73.7)$ & $2.7(5.1)$ \\
\hline PCMBS* & $2.7(2.8-2.7)$ & 145159 & 49479 & $11.2(2.2)$ & $93.6(54.6)$ & $10.6(30.1)$ \\
\hline $\mathrm{K}_{2} \mathrm{PtCl}_{2}^{*}$ & $2.9(3.0-2.9)$ & 108075 & 40112 & $12.4(2.5)$ & $95.0(78.1)$ & $8.6(24.9)$ \\
\hline $\mathrm{HgOAc}^{\star}$ & $2.6(2.7-2.6)$ & 158161 & 49295 & $16.6(1.9)$ & $82.6(31.1)$ & $7.3(25.1)$ \\
\hline SeMet $^{\star} \S$ (inflection) & $2.5(2.6-2.5)$ & 218666 & 130457 & $14.7(4.8)$ & $98.1(95.3)$ & $5.6(11.8)$ \\
\hline SeMet $^{\star}$ (maximum) & $2.5(2.6-2.5)$ & 218779 & 130517 & $13.7(4.8)$ & $98.2(96.9)$ & $5.9(12.9)$ \\
\hline SeMet $^{\star}$ (remote) & $2.5(2.6-2.5)$ & 218542 & 130802 & $13.1(4.8)$ & $97.7(92.9)$ & $6.0(13.1)$ \\
\hline \multicolumn{7}{|c|}{$\begin{array}{l}\text { * Partially occupied NADP }{ }^{+} \text {complex. } \\
\text { † Used as the native for multiple-isomorphous-replacment phasing of the partially occupied } \text { NADP }^{+} \text {complex. } \\
\text { * Fully occupied NADP } \\
\S \text { Complex. } \\
\text { U Used as the native for refinement of the partially occupied NADP }{ }^{+} \text {complex. } \\
\| \text { Bracketed numbers correspond to the data statistics in the final resolution shell. }\end{array}$} \\
\hline
\end{tabular}

Table 2 Refinement statistics

RMS, root mean square.

\begin{tabular}{|c|c|c|}
\hline & $\begin{array}{l}\text { Low-occupancy } \\
\text { NADP }^{+} \text {complex }\end{array}$ & $\begin{array}{l}\text { Full-occupancy } \\
\text { NADP }^{+} \text {complex }\end{array}$ \\
\hline No. of reflections used & 63932 & 92440 \\
\hline Resolution $(\AA)$ & $50.0-2.5$ & $50.0-2.1$ \\
\hline Protein atoms/tetramer & 15107 & 15039 \\
\hline Number of ligand atoms & 124 & 192 \\
\hline Occupancy of the ligand & 0.5 & 1.0 \\
\hline \multicolumn{3}{|l|}{ Average temperature factors $\left(\AA^{2}\right)$} \\
\hline Protein & 14.9 & 18.7 \\
\hline Ligand & 7.5 & 39.0 \\
\hline \multicolumn{3}{|l|}{ RMS temperature factors $\left(\AA^{2}\right)$} \\
\hline Main-chain bond (1-2 neighbours) & 1.4 & 0.9 \\
\hline Main-chain angle (1-3 neighbours) & 2.2 & 1.3 \\
\hline Side-chain bond & 2.4 & 1.5 \\
\hline Side-chain angle & 3.4 & 2.1 \\
\hline Number of solvent molecules & 413 & 652 \\
\hline$R$ & 0.206 & 0.215 \\
\hline$R_{\text {free }}$ & 0.239 & 0.246 \\
\hline Bond distances $(\AA)$ & 0.006 & 0.006 \\
\hline Bond angles $\left({ }^{\circ}\right)$ & 1.2 & 1.3 \\
\hline Dihedrals $\left({ }^{\circ}\right)$ & 22.3 & 22.5 \\
\hline Impropers $\left({ }^{\circ}\right)$ & 0.9 & 1.0 \\
\hline
\end{tabular}

made by Moore et al. [17] that there are different binding modes for the nicotinamide half of the cofactor.

The refinement statistics for both structures are shown in Table 2. The qualities of the structures were evaluated using the program package PROCHECK [30] and revealed no residues in the disallowed regions of the phi/psi plot. The co-ordinates have been deposited in the Protein Data Bank [31] with the accession number 1eyy for the partially occupied complex and 1ez0 for the fully occupied complex.

\section{RESULTS}

\section{Structure description}

The $V$. harveyi structure comprises four identical protein protomers per asymmetric unit related by three non-crystallographic 2-fold symmetry axes. The protomer is composed of three domains: a cofactor-binding domain consisting of residues 5-107 ( $\alpha 1-4), 138-255(\alpha \mathrm{A}-\mathrm{D}$ and $\beta \mathrm{A}-\mathrm{E})$ and $455-474(\beta 5$ and $\alpha 7)$; a catalytic domain comprising residues $256-454\left(\alpha \mathrm{A}^{\prime}-\mathrm{D}^{\prime}\right.$, $\beta \mathrm{A}^{\prime}-\mathrm{E}^{\prime}, \alpha 5-6$ and $\left.\beta 3-4\right)$ and an oligomerization domain made up of residues 108-137 $(\beta 1, \beta 2)$ and $475-508(\beta 5, \beta 7)$ (Figure 1, top panel). The numbering of the secondary-structure elements follows that described for betaine aldehyde dehydrogenase (ALDH9) [16]. Despite the low sequence similarity and differences in cofactor specificity amongst the family of aldehyde dehydrogenases the structures are all similar.

The N-terminus of the protein comprises $4 \alpha$-helices followed by a $\beta$-hairpin extension into the oligomerization domain from residues 107 to 138 . At residue 138 the chain enters the first strand, $\beta \mathrm{A}$, of a non-classical $\alpha / \beta$ Rossmann-type fold where only 5 of the $6 \beta$-strands are present. The last helix, $\alpha \mathrm{E}$ of the classical Rossmann fold, is missing in the structure and the last strand, $\beta \mathrm{F}$, has been replaced by $\beta 5$.

The catalytic domain adopts an $\alpha / \beta$ motif similar to that seen in the cofactor-binding domain; however, a number of insertions are present. The residues comprising $\alpha 5, \beta 3, \beta 4$ and $\alpha 6$ have been inserted between $\alpha \mathrm{B}^{\prime}$ and $\beta \mathrm{C}^{\prime}$ of the canonical Rossmann fold. A similar insertion has been reported in the structure of ALDH2 [14], whereas in ALDH9 the above insertion contains an extra helix between strands $\beta 3$ and $\beta 4$ [16]. As is observed in the cofactor-binding domain, the $\alpha \mathrm{E}^{\prime}$ helix and $\beta \mathrm{F}^{\prime}$ strand of the classical Rossmann fold are missing in the substrate-binding domain. Rather, after the $\beta \mathrm{E}^{\prime}$ strand the chain briefly re-enters the cofactor-binding domain, contributing a strand, $\beta 5$, and a short $\alpha$-helix, $\alpha 7$, and finally completes a further two strands in the oligomerization domain.

Aldehyde dehydrogenase from $V$. harveyi is a homodimer of $110 \mathrm{kDa}$ [32]. In the asymmetric unit of the crystal, the enzyme is arranged as a dimer of dimers. Within a dimer there are extensive intermolecular contacts mainly through the oligomerization domain and residues 235-243 of the $\alpha \mathrm{D}$ helix (Figure 1 , bottom panel). The area on each monomer that is involved in dimer formation is $3500 \AA^{2}$. The oligomerization domain is composed of a four-stranded mixed $\beta$-pleated sheet made up of strands $\beta 1, \beta 2, \beta 6$ and $\beta 7$. When viewed as a dimer this sheet structure is part of a larger $\beta$-sheet involving the substratebinding domain of the second protomer within the dimer. Intermolecular hydrogen-bonding contacts are made between main-chain atoms from residues $477-481$ of the $\beta 6$ strand in the oligomerization domain of one protomer and residues $432-435$ 

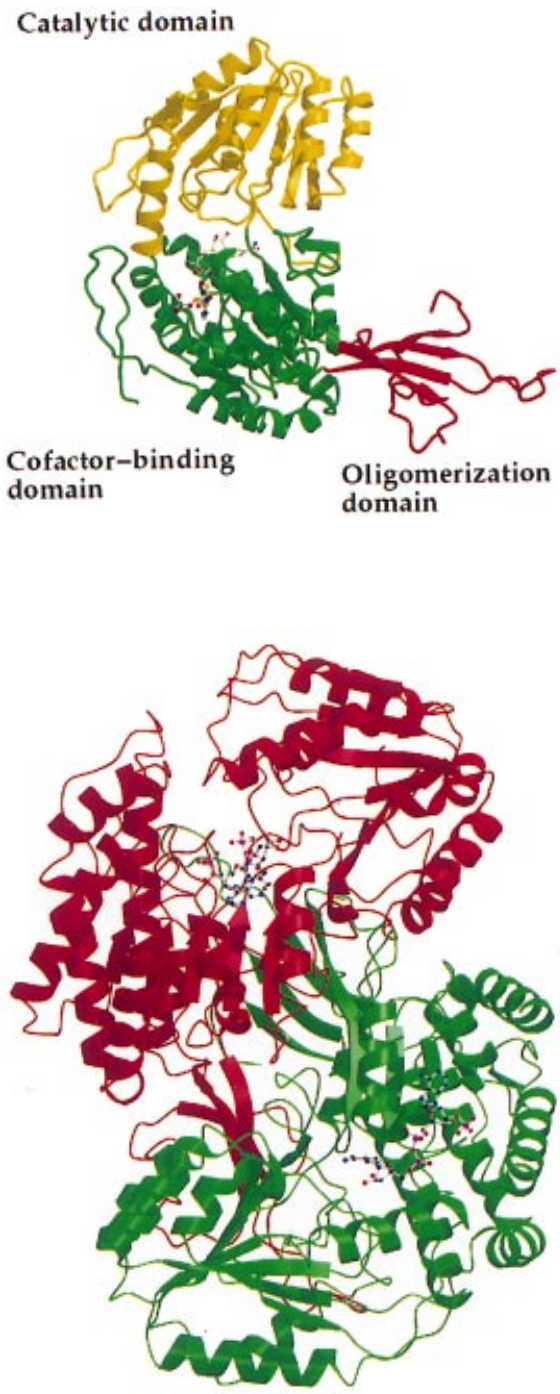
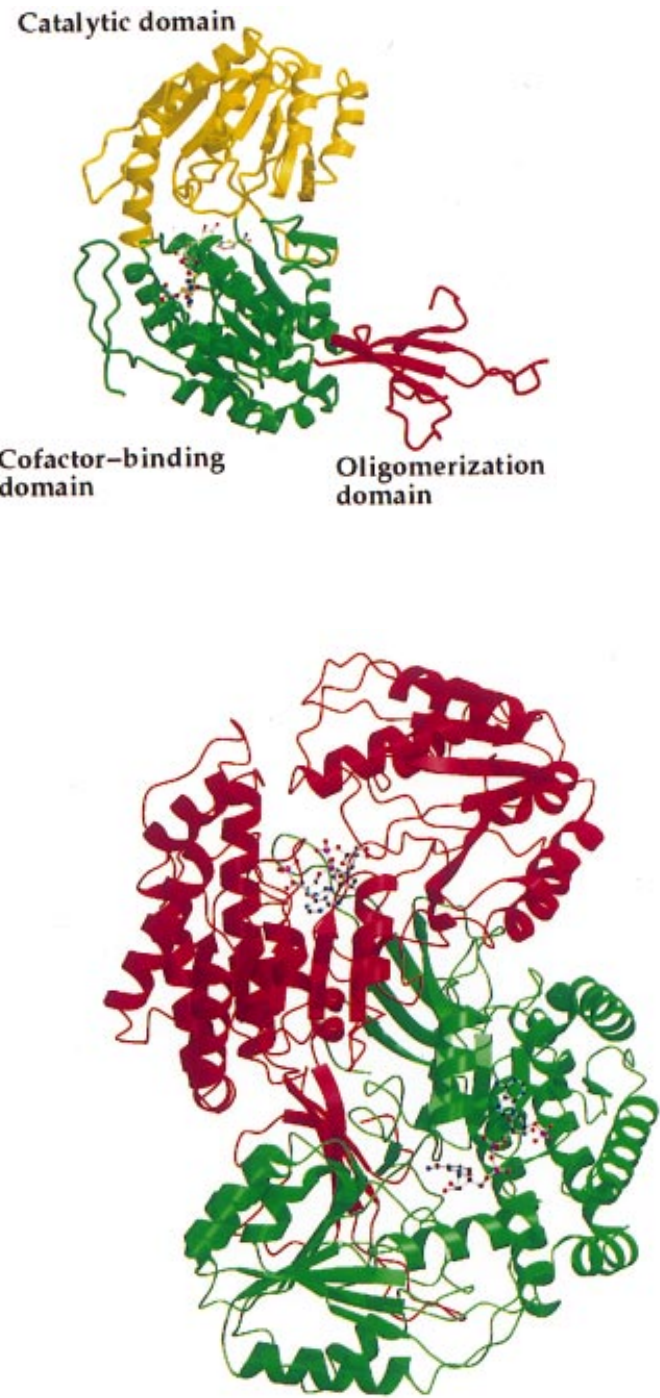

Figure 1 The structure of aldehyde dehydrogenase from $V$. harveyi

(Top panel) A ribbon representation showing the secondary-structure elements for a single protomer. The three domains are coloured differently and are labelled according to the description in the text. (Bottom panel) A ribbon representation showing the dimer of aldehyde dehydrogenase. The protomers within the dimer are coloured red and green. The NADP+ cofactor is shown as a ball-and-stick representation. The ribbon representation was made with the program MOLSCRIPT [41] and rendered using Raster 3D [42].

of the $\beta \mathrm{E}^{\prime}$ strand in the catalytic domain of the second protomer. These contacts result in the formation of an 11-stranded $\beta$ pleated sheet across the dimeric unit.

Other intermolecular contacts important in maintaining the dimeric unit include three salt-bridge interactions: Arg-44-Glu428, Arg-44-Glu-399 and Arg-47-Glu-427, all between the cofactor-binding domain of one protomer and the catalytic domain of the second protomer. This region forms an electrostatic surface with three arginine residues (Arg-44, Arg-47 and Arg-48) of one protomer packing against three glutamate residues (Glu399, Glu-427 and Glu-428) of the second protomer.

Interestingly, the $\alpha$-carbon atom of the final residue (508) in the model of one protomer lies only $5.8 \AA$ from that of the second protomer within a dimer. The sequence of the enzyme extends a further two residues; however, there is not sufficient electron density to model these final residues. The extension of the last two residues in each monomer would in effect lengthen the chain in order to complete a fifth $\beta$-strand in the oligo- merization domain. This would result in a 24 -stranded $\beta$-pleated sheet with 10 strands from the oligomerization domains and 14 strands from the catalytic domains within the dimeric unit.

Of all the structures of aldehyde dehydrogenase known to date, two are dimeric: the $V$. harveyi enzyme and ALDH3. The structures of the tetrameric forms of aldehyde dehydrogenase all reveal a packing of the three-stranded $\beta$-sheet in the oligomerization domain of one monomer with that of a second monomer in the tetramer. In the case of the dimeric proteins a number of structural features preclude the formation of the tetramer. The lack of a tetramer assembly for ALDH3 has been reported to be due to two factors: a rotational movement of the oligomerization domain, and the presence of an extended loop between residues 80 and 96 which would result in a collision with a neighbouring molecule within the tetramer [19]. In the $V$. harveyi enzyme the rotation of the oligomerization domain is not observed. Superposition of the cofactor-binding domains of aldehyde dehydrogenase shows that only in the case of ALDH3 is 

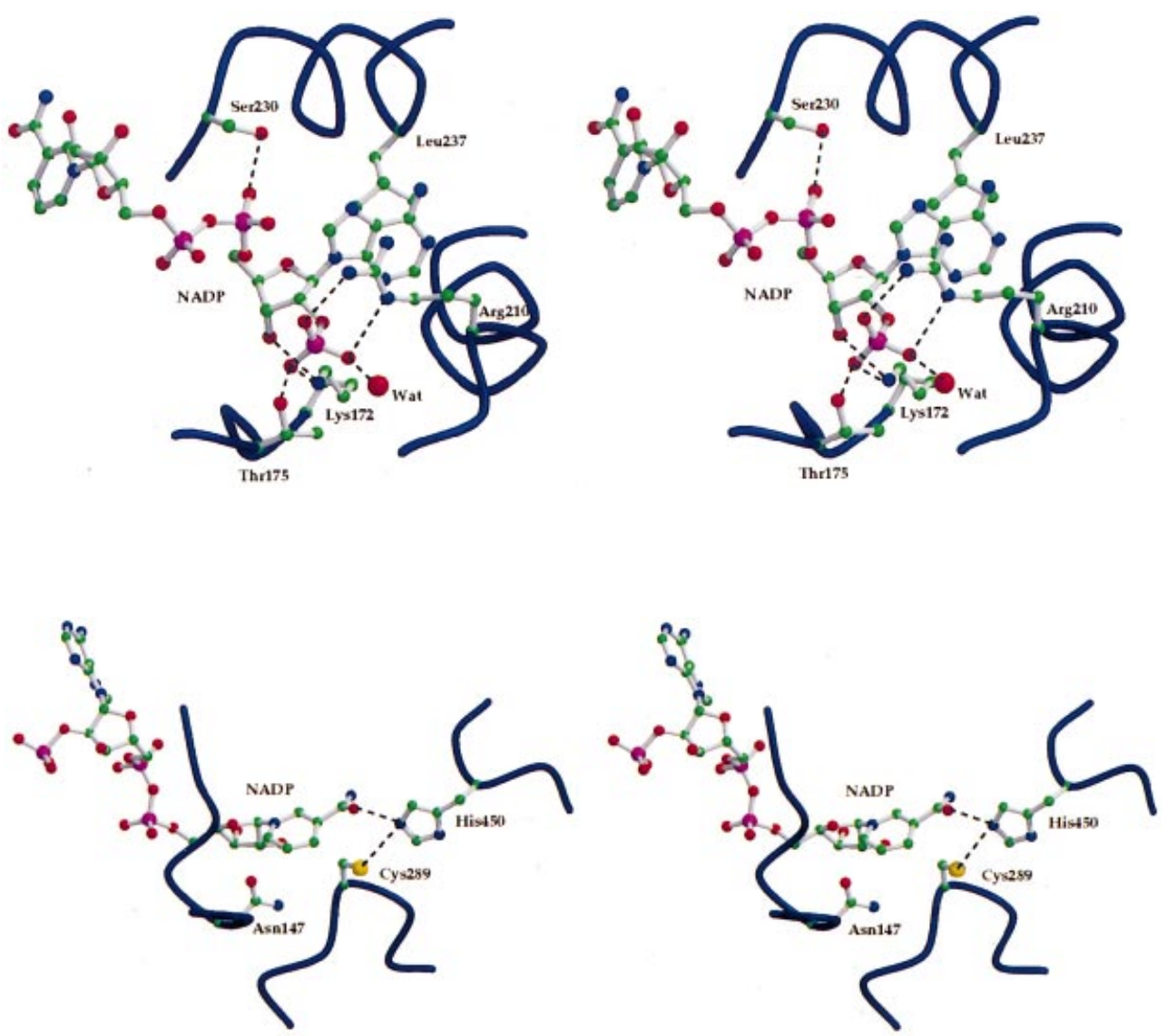

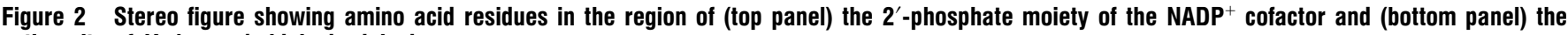
active site of $V$. harveyi aldehyde dehydrogenase

Regions of the main chain are shown as blue coils, the side chains as a ball-and-stick representation and hydrogen bonds as dashed lines.

the oligomerization domain significantly rotated despite the fact that both ALDH3 and the $V$. harveyi enzyme are dimeric species. The relative orientation of the oligomerization domain of the $V$. harveyi enzyme more closely matches that of the tetrameric forms of the protein. In the $V$. harveyi enzyme a C-terminal extension of residues 481-508 forms a loop around one face of the $\beta$-sheet in the oligomerization domain and a fourth $\beta$-strand. The formation of a tetramer is not possible due to the presence of this loop since it would cause significant steric clashes between two monomers within the tetramer. Although a C-terminal extension of 22 residues is also seen in the structure of ALDH3 this region of the chain forms a random coil interacting with the catalytic domain of the second subunit within the dimer [15]. Thus in the case of the two dimeric enzymes the C-terminal extensions are structurally very different and play different roles in terms of oligomerization.

\section{NADP $^{+}$-binding region}

In the structure of aldehyde dehydrogenase from $V$. harveyi reported here we see a well-defined electron density for the ADPP of the cofactor providing us with a clear view of the amino acid residues that interact with the $2^{\prime}$-phosphate of the cofactor. This enables us to compare the important molecular features that dictate cofactor specificity within the aldehyde dehydrogenase family of enzymes. The structure of an $\mathrm{NADP}^{+}$-dependent aldehyde dehydrogenase from $S$. mutans [19] and comparisons with the known $\mathrm{NAD}^{+}$-dependent forms has suggested key residues which are important for cofactor specificity. These residues include a lysine (Lys-177) which interacts with the 2'-phosphate group of $\mathrm{NADP}^{+}$and a threonine (Thr-180) which, due to its size, provides a pocket in which the phosphate group of NADP ${ }^{+}$ can bind.

In the $V$. harveyi enzyme we identify a number of key residues involved in interaction with the cofactor important for high cofactor specificity as well as the very low $K_{\mathrm{m}}$ seen for $\mathrm{NADP}^{+}$by this enzyme [1]. The guanidinium group of Arg-210 stacks over the adenine ring system of the cofactor, facilitating a pi stacking interaction (Figure 2, top panel). No other hydrogenbonding interactions are observed between the protein and the adenine ring system of the cofactor. A molecular surface construction of the $V$. harveyi enzyme reveals a cleft where the cofactor binds (Figure 3a). The side chain of Arg-210 acts like a 'gate' holding the adenine ring in place within the cleft. The structures of all other aldehyde dehydrogenases determined lack an arginine at this position and show that the cofactor-binding site lies in an open cleft along one side of the molecule. Figure 3(b) shows the surface in the region of the $\mathrm{NADP}^{+}$-binding cleft for the enzyme from $S$. mutans. The $\mathrm{NAD}^{+}$-specific forms of the enzyme all have an identical open cofactor-binding cleft to that seen for the $S$. mutans enzyme. The position of Arg-210 in the $V$. harveyi enzyme, and its observed interaction with the co- 


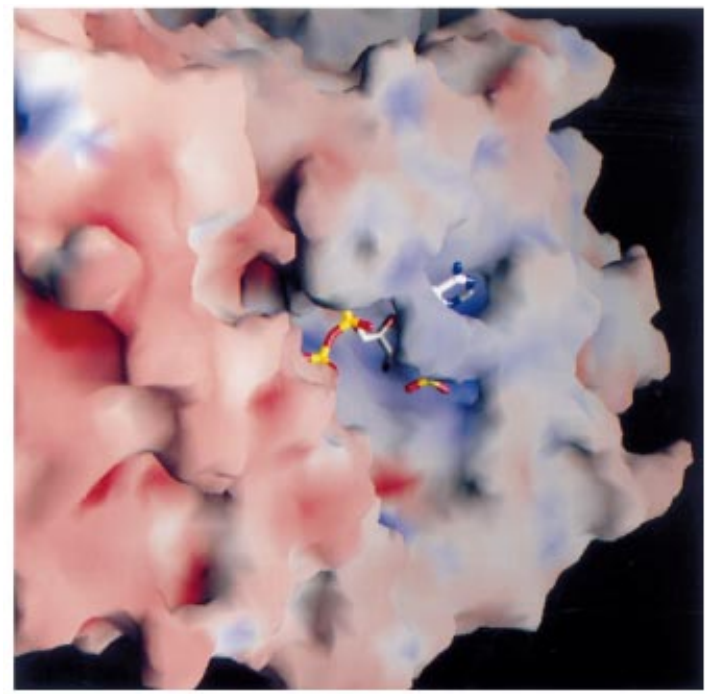

a)

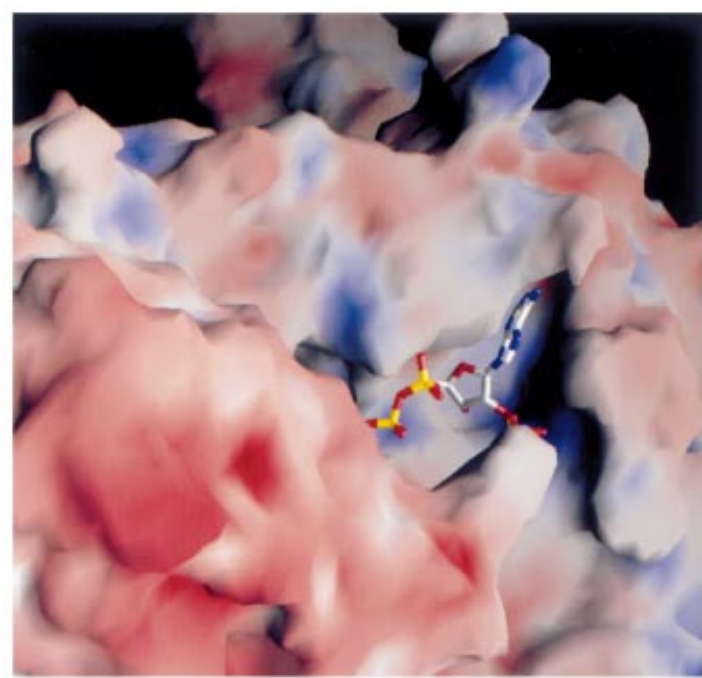

b)

Figure 3 Molecular surface representations of a portion of the cofactorbinding regions for aldehyde dehydrogenase from (a) V. harveyi and (b) S. mutans

The electrostatic potential has been mapped on to the surface between $-15.0 \mathrm{kT}$ (deep red) and $+15.0 \mathrm{kT}$ (deep blue). The $\mathrm{NADP}^{+}$cofactor is shown in stick representation.

factor, suggest that it may function to significantly improve cofactor affinity and may explain the very low $K_{\mathrm{m}}$ observed for $\mathrm{NADP}^{+}$in ALDH from $V$. harveyi.

Other interactions with the cofactor include extensive interactions with the $2^{\prime}$-phosphate group. Aside from a direct interaction with the hydroxy group of Thr-175, the side chains of Arg-210 and Lys-172 also make hydrogen-bond contacts with the oxygen atoms of the 2'-phosphate group (Figure 2, top panel). Other interactions include hydrogen bonds between the pyrophosphate group and the main-chain nitrogen and sidechain hydroxy group of Ser-230 that lies within the fingerprint region of the cofactor-binding domain.

Due to the weak electron density for the nicotinamide and ribose moieties of the $\mathrm{NADP}^{+}$in both the partially bound structure and the holoenzyme complex, we used the cofactor position in the structures of ALDH2 and ALDH9 to aid in modelling this region of the ligand in the $V$. harveyi enzyme structure. The refined nicotinamide and ribose groups have significantly higher temperature factors than observed for the adenine end of the cofactor, as expected considering the weak electron density for this region of the ligand. The 2'- and 3'hydroxy groups on the ribose moiety make weak hydrogenbond interactions with the carboxylate group of Glu-377. This glutamate is strictly conserved in all aldehyde dehydrogenases. Mutation of Glu-377 in the $V$. harveyi enzyme indicates that this residue is not involved in substrate binding or in acylation or deacylation but affects the hydride-transfer step of the reaction [12]. This suggests that the glutamate side chain may be involved in orienting the cofactor correctly for hydride transfer.

A hydrogen bond is observed between the carbonyl oxygen of the nicotinamide moiety of the cofactor and the side chain of His-450 (Figure 2, bottom panel). The side chain of Cys-289 is positioned near to NC-4 of the nicotinamide ring. In both the holoenzyme and the partially bound structures the electron density in the vicinity of the cysteine side chain indicates a possible alternate conformation. The modelled cysteine side chain $\left(\chi_{1}=51^{\circ}\right)$ places the thiol group within hydrogen-bonding distance of the amide oxygen atom of the nicotinamide moiety and close to the side chain of His-450. The alternate conformation $\left(\chi_{1} \approx-60^{\circ}\right)$ would position the thiol group within hydrogenbonding distance of the side chain of Asn-147. The temperature factor of the cysteine sulphur atom is $28 \AA^{2}$, significantly higher than the side chains of other residues in the immediate vicinity of Cys-289. This further suggests an increased mobility for this side chain.

A conserved basic residue, Glu-253, is positioned in the structure on the opposite face of the nicotinamide ring to that of Cys-289. This residue has been implicated as the base responsible for proton abstraction from Cys-289. However, in all reported structures of aldehyde dehydrogenase, this residue is positioned too far away from the cysteine side chain to abstract the thiol proton directly. Indeed, in the structure of $V$. harveyi aldehyde dehydrogenase we also found the glutamate side chain over $8 \AA$ away from Cys-289. In both the partially bound structure and the fully bound complex the electron density for the glutamate side chain is weak and the temperature factors are significantly higher than for other surrounding residues. This suggests that the glutamate side chain is flexible, similar to what has been observed for the side chain of Cys-289. In addition, comparison of the known structures of ALDH indicates that there is flexibility of the nicotinamide and ribose moieties. This conclusion has been made on the basis of the fact that in all aldehyde dehydrogenase structures the nicotinamide and ribose moieties are poorly defined in the electron density, and have high atomic temperature factors. Furthermore, the structure of ALDH1 is reported to have two conformations for the nicotinamide and ribose moieties of the $\mathrm{NAD}^{+}$cofactor [17]. One conformation is similar to the cofactor positions in ALDH2, ALDH9 and the $S$. mutans enzyme. The second conformation is more similar to that found in ALDH3 where the position of the nicotinamide ring is drawn back into the binding cleft and corresponds more closely to that of the ribose ring in $\mathrm{ALDH} 2, \mathrm{ALDH} 3$ and $V$. harveyi structures.

\section{DISCUSSION}

\section{Structural comparisons with other forms of aldehyde dehydrogenase}

Aldehyde dehydrogenase from $V$. harveyi differs from other forms of the enzyme due to its unique specificity and high affinity for $\mathrm{NADP}^{+}$. A structure determination of this $\mathrm{NADP}^{+}$-specific enzyme provides a basis for understanding the molecular features that control co-enzyme specificity and affinity. 
Structural comparisons of the $V$. harveyi enzyme with $\mathrm{NAD}^{+}$and $\mathrm{NADP}^{+}$-specific aldehyde dehydrogenases and with other $\mathrm{NADP}^{+}$-specific enzymes have been carried out. Differences in the co-enzyme-protein interactions are observed in the region of the $2^{\prime}$-phosphate group and these interactions have been compared with other aldehyde dehydrogenase enzymes and with a number of $\mathrm{NADP}^{+}$-specific enzymes. In the $\mathrm{NADP}^{+}$-specific aldehyde dehydrogenase from $V$. harveyi we observe two positively charged residues, Lys-172 and Arg-210, and one neutral residue, Thr-175, involved in hydrogen-bonding interactions with the 2'-phosphate group of the cofactor (Figure 2, top panel). Lys-172 is conserved amongst all aldehyde dehydrogenases and, in ALDH1, ALDH2 and ALDH9, the side chain makes a hydrogen-bond interaction with the 2 - and 3 '-hydroxy groups of the $\mathrm{NAD}^{+}$cofactor. In ALDH3 these interactions are not observed due to the altered binding mode of the cofactor. In aldehyde dehydrogenase from $S$. mutans the conserved lysine residue makes hydrogen-bond contacts with the 2 -phosphate group as well as the main-chain oxygen atoms of Pro-178 and Gly-208. In the $V$. harveyi enzyme the hydrogen bond to O-Gly173 (structurally equivalent position to Gly-208 in the S. mutans enzyme) is replaced by a hydrogen bond to a water molecule. This is due to the fact that the main chain in the loop regions from residue 206 to 211 in the $V$. harveyi structure have moved away from the ligand as compared with the identical region of the chain in the other aldehyde dehydrogenase structures. In contrast to the lysine residue, Thr-175 is unique to the $\mathrm{NADP}^{+}$specific forms of aldehyde dehydrogenase. In the $\mathrm{NAD}^{+}$-specific enzymes the equivalent residues at this position are Glu195 (ALDH1 and ALDH2), Glu-140 (ALDH3) and Pro-168 (ALDH9). The glutamate side chains contact the 2'- and 3'hydroxy groups on the adenosyl ribose ring of the $\mathrm{NAD}^{+}$ cofactor. This acidic residue is reported to be part of the consensus sequence for $\mathrm{NAD}^{+}$-binding proteins [33]. The adoption of a residue with a shorter side chain as well as a hydrogen-bond donor at this position of the $\mathrm{NADP}^{+}$-preferring enzymes appears to avoid the possible steric hindrance and electrostatic repulsion with the 2 '-phosphate oxygen group. Mutation of this residue to glutamate dramatically increases the $K_{\mathrm{m}}$ for NADP ${ }^{+}$and reduces the $K_{\mathrm{m}}$ for $\mathrm{NAD}^{+}$[34]. The arginine residue (Arg-210) is unique for the $V$. harveyi form of aldehyde dehydrogenase and its position over the adenine ring of the cofactor promotes pi stacking interactions between the two moieties. The guanidinium group of Arg-210 is also involved in a hydrogen-bond interaction with the $2^{\prime}$-phosphate group of the $\mathrm{NADP}^{+}$cofactor. The $S$. mutans structure does not have an arginine residue at the identical position. In addition, the $K_{\mathrm{m}}$ for $\mathrm{NADP}^{+}$in aldehyde dehydrogenase from $S$. mutans is $24.5 \mu \mathrm{m}$ [4], significantly higher than that observed for the $V$. harveyi enzyme, $1.4 \mu \mathrm{m}$ [1]. Thus the enhanced affinity for $\mathrm{NADP}^{+}$in the $V$. harveyi enzyme is correlated with the added pi stacking and hydrogen-bonding interactions between the cofactor and Arg-210. The presence of Lys-172 and Thr-175 provides cofactor specificity and Arg-210 contributes to high cofactor affinity. From this analysis we conclude that in the $V$. harveyi enzyme, Arg-210 is important for both $\mathrm{NADP}^{+}$specificity and high cofactor affinity through: (i) electrostatic interaction with the 2'-phosphate group of the $\mathrm{NADP}^{+}$cofactor and (ii) pi stacking interaction between the adenine ring system and the guanidinium side chain of the arginine residue. Furthermore, in the case of $\mathrm{NADP}^{+}$-dependent aldehyde dehydrogenases, the side chain of Thr-175 is also critical for cofactor specificity since it makes an important hydrogen bond with the 2 -phosphate group. The lysine residue appears to play a more important role in interaction with the $3^{\prime}$ hydroxy group of the cofactor since it is observed in both the
$\mathrm{NAD}^{+}$- and $\mathrm{NADP}^{+}$-specific forms of ALDH and is not structurally conserved in other $\mathrm{NADP}^{+}$-specific enzymes.

The pyrophosphate group of $\mathrm{NADP}^{+}$in the $V$. harveyi enzyme makes contact with the loop region between $\beta \mathrm{D}$ and $\alpha \mathrm{D}$. This is in contrast to what has been observed in the canonical nucleotidebinding proteins where the pyrophosphate group contacts the loop between $\beta \mathrm{A}$ and $\alpha \mathrm{A}$ and the consensus sequence consists of GXGXXG. In the $V$. harveyi enzyme the sequence in this loop region (229-234) is different from that of the consensus; however, it is rich in glycines (GSVGGG), therefore facilitating contact between the negatively charged phosphate group and the $\mathrm{N}$ terminal region of helix $\alpha \mathrm{D}$. Thus the negatively charged phosphate oxygen atoms are stabilized by the helix dipole [35].

\section{Catalytic mechanism}

In aldehyde dehyrogenase, the mechanism of catalysis involves the formation of an acyl enzyme intermediate upon attack of the carbonyl carbon of the aldehyde substrate by a cysteine residue in the active site. The intermediate then undergoes hydride transfer followed by deacylation. The active site consists of a funnel that extends from the surface of the enzyme to Cys-289, the active-site nucleophile. The side chain of Cys-289 is positioned in a hydrophobic pocket bounded by Phe-148, Phe-152, Phe-288, Phe-379, Leu-405 and Thr-290. Interestingly, in one conformation, the cysteine side chain makes close contact with the imidazole ring of His-450 and, in the second conformation, the cysteine side chain contacts Asn-147. All the residues, with the exception of His-450, are either completely conserved or similar within the family; the equivalent position to His-450 is a phenylalanine in the other aldehyde dehydrogenase structures.

The mechanism of catalysis calls for a basic residue to extract the proton from Cys-289 in order to increase its nucleophilicity for attack at the carbonyl carbon of the aldehyde substrate to form the hemithioacetal intermediate. Upon hydride transfer to the $\mathrm{NAD}(\mathrm{P})^{+}$cofactor a thioacylenzyme intermediate is formed. Finally this intermediate is hydrolysed through a baseactivated water molecule. On the basis of sequence similarity and mutagenesis experiments, the residue implicated to activate the thiolate is a glutamate and corresponds to Glu-253 in the $V$. harveyi enzyme [11,12,36]. Structural studies of all aldehyde dehydrogenases, however, have shown that this glutamate side chain is too far away to act directly as a base. In the case of the $V$. harveyi enzyme, the side chain of Glu-253 is located $8.7 \AA$ from that of Cys-289. Furthermore, the glutamate side chain is shielded from the cysteine residue by the nicotinamide ring of the cofactor. This suggests either that this glutamate is not directly involved in activation of the reactive cysteine or that its basic function is mediated either through another residue or through a water molecule and that the nicotinamide ring of the cofactor must move into position near the cysteine side chain only after proton abstraction occurs. This potential movement in the nicotinamide position may explain the poor density observed for this portion of the cofactor. The structures of ALDH2, ALDH3 and ALDH9 were all determined in the presence of $\mathrm{NAD}^{+}$; however, the temperature-factor refinement for the cofactor differed in each case. For ALDH 2 and ALDH9 the cofactor underwent group temperature-factor refinement; for ALDH9 the refined temperature factor for the cofactor was reported to be $61.7 \AA^{2}$, indicating a high degree of mobility. In the case of ALDH1 the cofactor underwent individual temperature-factor refinement. Finally for aldehyde dehydrogenase from $S$. mutans the cofactor is well refined and has relatively low temperature factors. In our refined model of the holoenzyme, individual temperature-factor 
refinement was also carried out. Analysis of the $\mathrm{NADP}^{+}$ligand reveals that for both ALDH1 and the $V$. harveyi enzyme the individual atomic temperature factors for the nicotinamide portion of the cofactor are significantly higher than those of the adenine and adenosyl ribose moieties. This observation, as well as the poorly defined electron density for the nicotinamide moiety, indicates that the nicotinamide portion of the ligand may be highly mobile. These results further support the suggestion made by Moore et al. [17] that the nicotinamide and ribose portions of the ligand undergo a conformational change. Furthermore, recent tryptophan fluorescence studies by Marchal and Branlant have shown that the enzyme from $S$. mutans undergoes a conformational change upon cofactor binding [4]. Further analysis of key active-site residues (Glu-253 and Cys289) also indicates a high degree of mobility, suggesting that these side chains may move significantly in a concerted fashion to the movement of the nicotinamide ring. The cofactor may be positioned away from the active-site cysteine residue in order to facilitate proton abstraction by Glu-253 (possibly mediated through a water molecule), and then move into position near to the cysteine residue upon formation of the thiohemiacetal intermediate in order to allow hydride transfer to occur.

As an alternative to the proposed role of Glu-253 in thiolate activation we observe a histidine residue (His-450) in close proximity to the reactive cysteine group (Figure 2, bottom panel). Although this histidine is not observed in the other aldehyde dehydrogenase structures (a phenylalanine is seen at the identical position in other aldehyde dehydrogenases) it is seen in the structure of phosphorylating glyceraldehyde-3-phosphate dehydrogenase [37]. In this enzyme the histidine residue (His176 ) is the base activating the cysteine (Cys-149) for nucleophilic attack [38]. Although the $V$. harveyi enzyme is not known to be a phosphorylating aldehyde dehydrogenase it may be that the mechanism of cysteine activation is similar for the two enzymes and thus different from other forms of aldehyde dehydrogenase. Mutagenesis and kinetic analysis of this histidine residue will confirm its catalytic role.

Positioning of a substrate molecule into the holoenzyme near to Cys-289 places the carbonyl oxygen of the aldehyde substrate within hydrogen-bonding distance of the amide group of Asn147. This residue is conserved amongst the structures of aldehyde dehydrogenases determined so far. Indeed, amino acid alignments of 145 aldehyde dehydrogenase-related enzymes show that this residue is conserved in 143 cases [39]. This structural and sequence conservation indicates that the asparagine residue has a critical role to play in the enzyme mechanism and its position suggests that it may act to stabilize the oxyanion formed upon nucleophilic attack of the substrate [14]. Interestingly the density for the alternative conformation of the cysteine side chain places the sulphur within hydrogen-bonding distance of the amide nitrogen of Asn-147. This suggests that, in the absence of the aldehyde substrate, the thiolate may be stabilized by the amide group of Asn-147.

Further structural analysis of the ternary complex model suggests that the hydride transfer would occur on to the A-face of the nicotinamide ring, as has also been suggested for ALDH2 [14] and ALDH9 [16] on the basis of structural interpretation. This supports the stereospecificity studies carried out on different isozymes of aldehyde dehyrogenase enzyme which have shown that the hydride transfer does occur on to the A-face of the nicotinamide ring [40]. Further studies to test the model await structural characterization of the ternary complex of aldehyde dehyrogenase-NADP ${ }^{+}$-substrate.

In summary, we have determined the first crystal structure of an $\mathrm{NADP}^{+}$-specific form of aldehyde dehydrogenase in the presence of the cofactor. The structure adopts a non-classical $\alpha / \beta$ Rossmann fold similar to that observed in other aldehyde dehydrogenase structures. A comparison with the $\mathrm{NAD}^{+}$-specific forms of the enzyme reveals important features that mediate cofactor specificity as well as high affinity. The residues implicated in cofactor binding and in the catalytic mechanism will be tested further by mutagenesis, kinetic and structural analyses.

We thank N. Croteau for crystallization of the protein. This work was supported by grants from the Medical Research Council of Canada (MT13341 to A. V. and MT4314 to E.M.), the Natural Sciences and Engineering Research Council of Canada (194585-98 to A.V.), the Fonds de la recherche en santé du Québec (Chercheurboursier Salary Support Award to A. V.) and the Medical Research Council of Canada (studenship to L.Z.)

\section{REFERENCES}

1 Byers, D. and Meighen, E. (1984) Vibrio harveyi aldehyde dehydrogenase. Partial reversal of aldehyde oxidation and its possible role in the reduction of fatty acids for the bioluminescence reaction. J. Biol. Chem. 259, 7109-7114

2 Lindahl, R. (1992) Aldehyde dehydrogenases and their role in carcinogenesis. Crit. Rev. Biochem. 27, 283-355

3 Jones, D. G., Brennan, M. D., Hempel, J. and Lindahl, R. (1988) Cloning and complete nucleotide sequence of a full length CDNA encoding a catalytically functional tumour-associated aldehyde dehydrogenase. Proc. Nat. Acad. Sci. U.S.A. 85, 1782-1786

4 Marchal, S. and Branlant, G. (1999) Evidence for the chemical activation of essential Cys-302 upon cofactor binding to nonphosphorylating glyceraldehyde 3-phosphate dehydrogenase from Streptococcus mutans. Biochemistry 38, 12950-12958

5 Hempel, J., Nicholas, H. and Lindahl, R. (1993) Aldehyde dehydrogenases: widespread structural and functional dispersity within a shared framework. Protein Sci. 2, 1892-1900

6 Vedadi, M., Szittner, R., Smillie, L. and Meighen, E. (1995) Involvement of cysteine 289 in the catalytic activity of an NADP ${ }^{+}$-specific fatty aldehyde dehydrogenase from Vibrio harveyi. Biochemistry 34, 16725-16732

7 Yin, S. J., Wang, S. L., Leao, C. S. and Jörnvall, H. (1993) Human high $K_{M}$ aldehyde dehydrogenases (ALDH3): molecular, kinetic and structural features, in Enzymology and Molecular Biology of Carbonyl Metabolism, vol. 4 (Weiner, J., Wermuth, B. and Crabb, D. W., ed.), pp. 87-98, Plenum Press, New York

8 Yin, S. J., Wang, M. F., Han, C. L. and Wang, S. L. (1995) Substrate binding pocket structure of human aldehyde dehydrogenases. A substrate specificity approach. Adv. Exp. Med. Biol. 372, 9-16

9 Vedadi, M., Croteau, N., Delarge, M., Vrielink, A. and Meighen, E. (1996) Structural and function studies of a NADP ${ }^{+}$-specific aldehyde dehydrogenase from the luminescent marine bacterium Vibrio harveyi, in Enzymology and Molecular Biology of Carbonyl Metabolism, vol. 414 (Weiner, J., Wermuth, B. and Crabb, D. W., ed.) pp. 269-275, Plenum Press, New York

10 Farrés, J., Wang, T. T. Y., Cunningham, S. J. and Weiner, H. (1995) Investigation of the active site cysteine residue of rat liver mitochondrial aldehyde dehydrogenase by site-directed mutagenesis. Biochemistry 34, 2592-2598

11 Wang, $X$. and Weiner, $H$. (1995) Involvement of glutamate 268 in the active site of human liver mitochondrial (class 2) aldehyde dehydrogenase as probed by sitedirected mutagenesis. Biochemistry 34, 237-243

12 Vedadi, M. and Meighen, E. (1997) Critical glutamic acid residues affecting the mechanism and nucleotide specificity of Vibrio harveyi aldehyde dehydrogenase. Eur. J. Biochem. 246, 698-704

13 Vedadi, M., Vrielink, A. and Meighen, E. (1997) Involvement of conserved glycine residues, 229 and 234, of Vibrio harveyi aldehyde dehydrogenase in activity and nucleotide binding. Biochem. Biophys. Res. Commun. 238, 448-451

14 Steinmetz, C. G., Xie, P., Weiner, H. and Hurley, T. D. (1997) Structure of mitochondrial aldehyde dehydrogenase: the genetic component of ethanol aversion. Structure 5, 701-711

15 Liu, Z. J., Sun, Y. J., Rose, J., Chung, Y. J., Hsiao, C. D., Chang, W. R., Kuo, I., Perozich, J., Lindahl, R., Hempel, J. and Wang, B.-C. (1997) The first structure of an aldehyde dehdrogenase reveals novel interactions between NAD and the Rossmann fold. Nat. Struct. Biol. 4, 317-326

16 Johansson, K., El-Ahmad, M., Ramaswamy, S., Helmqvist, L., Jörnvall, H. and Eklund, H. (1998) Structure of betaine aldehyde dehydrogenase at $2.1 \AA$ resolution. Protein Sci. 7, 2106-2117

17 Moore, S. A., Baker, H. M., Blythe, T. J., Kitson, K. E., Kitson, T. M. and Baker, E. N. (1998) Sheep liver cytosolic aldehyde dehydrogenase: the structure reveals the basis for the retinal specificity of class 1 aldehyde dehydrogenases. Structure $\mathbf{6}$, $1541-1551$ 
18 Lamb, A. L. and Newcomer, M. E. (1999) The structure of retinal dehydrogenase type II at $2.7 \AA$ resolution: implications for retinal specificity. Biochemistry 38, 6003-6011

19 Cobessi, D., Tête-Favier, F., Marchal, S., Azza, S., Branlant, G. and Aubry, A. (1999) Apo and holo crystal structures of an NADP-dependent aldehyde dehydrogenase from Streptococcus mutans. J. Mol. Biol. 290, 161-173

20 Croteau, N., Vedadi, M., Delarge, M., Meighen, E., Abu-Abed, M., Howell, P. L. and Vrielink, A. (1996) Crystallization and preliminary X-ray analysis of aldehyde dehydrogenase from Vibrio harveyi. Protein Sci. 5, 2130-2132

21 Otwinowski, Z. (1993) in Proceedings of the CCP4 Suite Study Weekend: Data Collection and Processing (Sawyer, L., Isaacs, N. and Bailey, S., eds.), pp. 56-62, SERC Daresbury Laboratory, Warrington

22 Minor, W. (1993) XDISPLAYF Program, Purdue University

23 Collaborative Computational Project, No. 4 (1994) The CCP4 suite: programs for protein crystallography. Acta Crystallogr. D50, 760-763

24 Hendrickson, W. A., Horton, J. R. and LeMaster, D. M. (1990) Selenomethionyl proteins produced for analysis by multiwavelength anomalous diffraction (MAD): a vehicle for direct determination of three-dimensional structure. EMBO 9, 1665-1672

25 Ramakrishnan, V. and Biou, V. (1997) Treatment of multiwavelength anomalous diffraction data as a special case of multiple isomorphous replacement. Methods Enzymol. 276, 538-557

26 Jones, T. A., Zou, J.-Y., Cowan, S. W. and Kjeldgaard, M. (1991) Improved methods for building protein models in electron density maps and the location of errors in these models. Acta Crystallogr. A47, 110-119

27 Jones, T. A. (1992) A yaap asap ${ }^{\circ} \#^{\star}$ ? A set of averaging programs, in Molecular Replacement (Dodson, E. J., Gover, S. and Wolf, W., eds.), pp. 91-105, SERC Daresbury Laboratory, Warrington

28 Kleywegt, G. J. and Read, R. J. (1997) Not your average density. Structure 5, 1557-1569

29 Rice, L. M. and Brünger, A. T. (1994) Torsion angle dynamics: reduced variable conformation sampling enhances crystallographic structure refinement. Proteins Struct. Funct. Genet. 19, 277-290

30 Laskowski, R. A., MacArthur, M. W., Moss, D. S. and Thornton, J. M. (1993) PROCHECK: a program to check the stereochemical quality of protein structures. J. Appl. Cryst. 26, 283-291

Received 22 November 1999/14 April 2000; accepted 22 May 2000
31 Bernstein, F. C., Koetzle, T. F., Williams, G. J., Meyer, E. E. J., Brice, M. D., Rodgers, J. R., Kennard, 0., Shimanouchi, R. and Tasumi, M. (1977) The Protein Data Bank: a computer-based archival file for macromolecular structures. J. Mol. Biol. 112 535-542

32 Bognar, A. and Meighen, E. A. (1978) An induced aliphatic aldehyde dehydrogenase from the bioluminescent bacterium Beneckea harveyi, purification and properties. J. Biol. Chem. 253, 446-450

33 Eklund, H., Samama, J. P. and Jones, T. A. (1984) Crystallographic investigations of nicotinamine adenine dinucleotide binding to horse liver alcohol dehydrogenase. Biochemistry 23, 5982-5996

34 Zhang, L., Ahvazi, B., Szittner, R., Vrielink, A. and Meighen, E. (1999) Change of nucleotide specificity and enhancement of catalytic efficiency in single point mutants of Vibrio harveyi aldehyde dehydrogenase. Biochemistry 38, 11440-11447

35 Wierenga, R. K., Terpstra, P. and Hol, W. G. J. (1986) Prediction of the occurrence of the ADP-binding $\beta \alpha \beta$ fold in protein, using an amino acid fingerprint. J. Mol. Biol. 187, 101-107

36 Abriola, D. P., Fields, R., Stein, S., Mackerell, J. A. D. and Pietruszko, R. (1987) Active site of human liver aldehyde dehydrogenase. Biochemistry 26, 5679-5684

37 Skarzynski, T., Moody, P. C. and Wonacott, A. J. (1987) Structure of hologlyceraldehyde-3-phosphate dehydrogenase from Bacillus stearothermophilus at $1.8 \AA$ resolution. J. Mol. Biol. 193, 171-187

38 Harris, J. I. and Waters, M. (1976) Glyceraldehyde-3-phosphate dehydrogenase. In The Enzymes, vol. 13C (Boyer, P. D., ed.), pp. 1-49, Academic Press, New York

39 Perozich, J., Nicholas, H., Wang, B.-C., Lindahl, R. and Hempel, J. (1999) Relationships within the aldehyde dehydrogenase extended family. Protein Sci. 8 $137-146$

40 Jones, K. H., Lindahl, R., Baker, D. C. and Timkovich, R. (1987) Hydride transfer sterospecificity of rat liver aldehyde dehydrogenases. J. Biol. Chem. 262 10911-10913

41 Kraulis, P. (1991) MOLSCRIPT: a program to produce both detailed and schematic plots of protein structures. J. Appl. Crystallogr. 24, 946-950

42 Merritt, E. A. and Murphy, M. E. P. (1994) Raster3D version 2.0. A program for photorealistic molecular graphics. Acta Crystallogr. D50, 869-873 